\title{
On the Fourier-Transformed Boltzmann Equation with Brownian Motion
}

\section{Yong-Kum Cho and Eunsil Kim}

Department of Mathematics, College of Natural Science, Chung-Ang University, 84 Heukseok-Ro, Dongjak-Gu, Seoul 156-756, Republic of Korea

Correspondence should be addressed to Yong-Kum Cho; ykcho@cau.ac.kr

Received 17 July 2014; Accepted 16 September 2014

Academic Editor: Xiao-Jun Yang

Copyright (C) 2015 Y.-K. Cho and E. Kim. This is an open access article distributed under the Creative Commons Attribution License, which permits unrestricted use, distribution, and reproduction in any medium, provided the original work is properly cited.

We establish a global existence theorem, and uniqueness and stability of solutions of the Cauchy problem for the Fouriertransformed Fokker-Planck-Boltzmann equation with singular Maxwellian kernel, which may be viewed as a kinetic model for the stochastic time-evolution of characteristic functions governed by Brownian motion and collision dynamics.

\section{Introduction}

In this paper, we consider the Cauchy problem for the spacehomogeneous Fokker-Planck-Boltzmann equation which takes the form

$$
\begin{array}{r}
\partial_{t} f(v, t)=Q(f, f)(v, t)+v \Delta f(v, t) \\
\text { for }(v, t) \in \mathbb{R}^{3} \times(0, \infty), \\
f(v, 0)=f_{0}(v) \quad \text { for } v \in \mathbb{R}^{3} .
\end{array}
$$

Here, the diffusion constant $v \geq 0, f_{0}$ is a nonnegative initial datum and $Q(f)$ stands for the collision term defined as

$$
\begin{aligned}
& Q(f, f)(v) \\
& \quad=\int_{\mathbb{R}^{3}} \int_{\mathbb{S}^{2}} b(\mathbf{k} \cdot \sigma)\left[f\left(v^{\prime}\right) f\left(v_{*}^{\prime}\right)-f(v) f\left(v_{*}\right)\right] d \sigma d v_{*}
\end{aligned}
$$

for each scalar-valued function $f$ on $\mathbb{R}^{3}$ where

$$
\begin{gathered}
v^{\prime}=\frac{v+v_{*}}{2}+\frac{\left|v-v_{*}\right|}{2} \sigma, \\
v_{*}^{\prime}=\frac{v+v_{*}}{2}-\frac{\left|v-v_{*}\right|}{2} \sigma, \\
\mathbf{k}=\frac{v-v_{*}}{\left|v-v_{*}\right|},
\end{gathered}
$$

the collision kernel $b$ is a nonnegative function on $[-1,1]$, and $d \sigma$ denotes the area measure on the unit sphere $\mathbb{S}^{2}$.

In kinetic theory of a rarefied gas, the Fokker-PlanckBoltzmann equation (1) models the single-particle distribution function $f$ of its molecules which evolve under binary and elastic collision dynamics as well as Brownian motion (see below). Each pair $\left(v^{\prime}, v_{*}^{\prime}\right)$ represents the postcollision velocities of two molecules colliding with velocities $\left(v, v_{*}\right)$.

The collision kernel $b$ is an implicitly-defined function which represents a specific type of collision dynamics in terms of the deviation angle $\theta$ defined by $\cos \theta=\mathbf{k} \cdot \sigma$. In a physically relevant model known as the Maxwellian kernel, it is customary to assume that $b(\cos \theta)$ is supported in $[0, \pi / 2]$, bounded away from $\theta=0$, but develops a singularity at $\theta=0$ in the form

$$
b(\cos \theta) \sin \theta \sim \theta^{-3 / 2} \text { as } \theta \longrightarrow 0+,
$$


which accounts for grazing collisions in the long-range interactions.

The Maxwellian kernel is a special instance of

$$
B=\left|v-v_{*}\right|^{\lambda} b(\cos \theta) \quad(-3<\lambda \leq 2),
$$

known as the collision kernel of inverse-power potential type, and we refer to Villani's review paper [1] for more details. Besides the physically relevant assumption (4) on $b$, a simplified one is that

$$
\|b\|_{L^{1}\left(\mathbb{S}^{2}\right)}=2 \pi \int_{0}^{\pi / 2} b(\cos \theta) \sin \theta d \theta<+\infty,
$$

referred to as Grad's angular cutoff assumption.

The inhomogeneous Fokker-Planck-Boltzmann equation reads

$$
\partial_{t} f+v \cdot \nabla_{x} f=Q(f, f)+v \Delta_{v} f \quad \text { in } \mathbb{R}^{3} \times \mathbb{R}^{3} \times(0, \infty)
$$

for the unknown density $f=f(x, v, t)$, where the space variable $x \in \mathbb{R}^{3}$ stands for the position. In the case when the collision kernel $B$ takes form (5) and the angular part $b$ satisfies certain cutoff assumption of type (6), let us mention some of the earlier works on the Cauchy problem for (7). In the small perturbations of the vacuum state, a global existence result is obtained by Hamdache [2]. In the context of renormalized solutions, global existence and stability of solutions with large data are established by DiPerna and Lions [3]. In the linearized context around the global Maxwellian $M(v)=(2 \pi)^{-3 / 2} \exp \left(-|v|^{2} / 2\right)$, global existence or asymptotic behavior of solutions is investigated by Li and Matsumura [4], Xiong et al. [5], and Zhong and Li [6]. We also refer to Li [7] for the diffusive property of solutions and further references cited in the aforementioned work.

As for the homogeneous Fokker-Planck-Boltzmann equation, we are aware only of results of Goudon [8] for the global existence of a weak solution in the case when the collision kernel is given by (5) with $-3<\lambda<-2$ and $b$ satisfies a singular condition of type (4). For the homogeneous Boltzmann equation, however, more extensive results are available. We refer to Arkeryd [9, 10], Goudon [8], and Villani [11] and to the references cited therein.

We recall that the Fourier transform of a complex Borel measure $\mu$ on $\mathbb{R}^{3}$ is defined by

$$
\widehat{\mu}(\xi)=\int_{\mathbb{R}^{3}} e^{-i \xi \cdot v} d \mu(v) \quad\left(\xi \in \mathbb{R}^{3}\right),
$$

which extends to any tempered distribution on $\mathbb{R}^{3}$ via the usual functional pairing relations. If $\mu$ is a probability measure, that is, a nonnegative Borel measure with unit mass, $\widehat{\mu}$ is said to be a characteristic function.

From a probability theory point of view, Cauchy problem (1), with an initial probability density $f_{0}$, could be considered as a governing equation for the time-evolution of a family of probability densities $\{f(\cdot, t)\}_{t>0}$ and, hence, it is natural to study the problem on the Fourier transform side for it is fundamental in probability theory to investigate a probability distribution through its characteristic function.

In [12], Bobylev discovered a remarkably simple formula for the Fourier transform of the collision term which reads

$$
\begin{gathered}
{[Q(f, f)]-(\xi)} \\
=\int_{\mathbb{S}^{2}} b\left(\frac{\xi \cdot \sigma}{|\xi|}\right)\left[\widehat{f}\left(\xi^{+}\right) \widehat{f}\left(\xi^{-}\right)-\widehat{f}(\xi) \widehat{f}(0)\right] d \sigma, \\
\xi^{+}=\frac{\xi+|\xi| \sigma}{2}, \quad \xi^{-}=\frac{\xi-|\xi| \sigma}{2}
\end{gathered}
$$

for each nonzero $\xi \in \mathbb{R}^{3}$. To simplify, we introduce the Boltzmann-Bobylev operator $\mathscr{B}$ defined by

$$
\mathscr{B}(\phi)(\xi)=\int_{\mathbb{S}^{2}} b\left(\frac{\xi \cdot \sigma}{|\xi|}\right)\left[\phi\left(\xi^{+}\right) \phi\left(\xi^{-}\right)-\phi(\xi) \phi(0)\right] d \sigma
$$

for each complex-valued function $\phi$ on $\mathbb{R}^{3}$. In view of Bobylev's formula, the Fourier-transformed version of (1) takes the form

$$
\begin{array}{r}
\left(\partial_{t}+\nu|\xi|^{2}\right) \phi(\xi, t)=\mathscr{B}(\phi)(\xi, t) \\
\text { for }(\xi, t) \in \mathbb{R}^{3} \times(0, \infty), \\
\phi(\xi, 0)=\phi_{0}(\xi) \quad \text { for } \xi \in \mathbb{R}^{3},
\end{array}
$$

which is equivalent to the integral equation

$$
\phi(\xi, t)=e^{-\nu|\xi|^{2} t} \phi_{0}(\xi)+\int_{0}^{t} e^{-\nu|\xi|^{2}(t-\tau)} \mathscr{B}(\phi)(\xi, \tau) d \tau,
$$

provided that differentiation under the integral sign was permissible.

In the theory of stochastic processes, a Markov process $\left\{X_{t}\right\}_{t \geq 0}$ in any Euclidean space $\mathbb{R}^{n}$, with stationary independent increments, for which the characteristic functions of its continuous transition probability densities are given by the Gaussian family $\left\{e^{-|\xi|^{2} t}\right\}_{t \geq 0}$ is known as Brownian motion or the symmetric stable Lévi process of index 2 (see [13]). Hence, Cauchy problem (11) may be viewed as a kinetic model for the stochastic time-evolution of characteristic functions governed by Brownian motion and Maxwellian collision dynamics. For more detailed interpretations and motivations, we refer to the inspiring paper [14] of Bisi et al. which deals with Cauchy problem (11) in the inelastic setting.

In this paper, we are concerned about global existence and uniqueness and stability of solutions of Cauchy problem (11) in the space of characteristic functions. Before proceeding further, let us describe briefly some of the earlier works about the Cauchy problem for the corresponding Fouriertransformed Boltzmann equation:

$$
\begin{gathered}
\partial_{t} \phi(\xi, t)=\mathscr{B}(\phi)(\xi, t) \\
\text { for }(\xi, t) \in \mathbb{R}^{3} \times(0, \infty), \\
\phi(\xi, 0)=\phi_{0}(\xi) \quad \text { for } \xi \in \mathbb{R}^{3}
\end{gathered}
$$


for which the Maxwellian kernel $b$ is assumed to satisfy the singular or noncutoff condition as described in (4).

(a) It is Pulvirenti and Toscani [15] who first established a global existence of solution to (13) on the space of characteristic functions $\phi$ satisfying

$$
\phi(0)=1, \quad \nabla \phi(0)=0, \quad \Delta \phi(0)=-3 .
$$

They also proved uniqueness and stability of solutions in terms of Tanaka's functionals related with probabilistic Wasserstein distance.

(b) In [16], Toscani and Villani proved uniqueness and stability, on the same solution space, with respect to the Fourier-based metric $d_{2}$ which is a particular case of

$$
d_{\alpha}(f, g)=\|\phi-\psi\|_{\alpha}=\sup _{\xi \in \mathbb{R}^{3}} \frac{|\phi(\xi)-\psi(\xi)|}{|\xi|^{\alpha}}
$$

for each $\alpha \geq 0$ where $\phi=\widehat{f}$ and $\psi=\widehat{g}$ (see also [17] for the properties of Fourier-based metrics and their applications to the Boltzmann and FokkerPlanck-Boltzmann equations in the inelastic setting).

(c) In [18], Bobylev and Cercignani constructed an explicit class of self-similar solutions whose probability densities possess infinite energy for all time. Specifically, they exhibited a class of characteristic functions $\Phi(\xi, t)$ satisfying (13) and $\Delta \Phi(0, t)=-\infty$ for all $t \geq 0$.

(d) In [19], Cannone and Karch established global existence and uniqueness and stability of solutions on the space $\mathscr{K}^{\alpha}$, to be explained below, which turns out to be larger than the solution space of Pulvirenti and Toscani and closely related with infinite energy solutions. In [20], Morimoto improved their work by weakening the assumptions on the kernel and providing another proof of uniqueness.

As to Cauchy problem (11), our aim is to establish global existence and uniqueness and stability of solutions on the space introduced by Cannone and Karch [19]. Following their notation, let $\mathscr{K}$ be the set of all characteristic functions on $\mathbb{R}^{3}$. For $0<\alpha \leq 2$, let

$$
\mathscr{K}^{\alpha}=\left\{\phi \in \mathscr{K}:\|\phi-1\|_{\alpha}=\sup _{\xi \in \mathbb{R}^{3}} \frac{|\phi(\xi)-1|}{|\xi|^{\alpha}}<+\infty\right\} .
$$

While $\mathscr{K}^{\alpha}$ is not a vector space, it is a complete metric space with respect to the Fourier-based metric $d_{\alpha}$ defined in (15) (for the proofs and further properties, see [19]). As a monotonically indexed family, the embedding

$$
\{1\} \subset \mathscr{K}^{\beta} \subset \mathscr{K}^{\alpha} \subset \mathscr{K}
$$

holds for $0<\alpha \leq \beta \leq 2$. Any characteristic function $\phi$ satisfying (14) clearly belongs to $\mathscr{K}^{2}$. More extensively, it can be trivially verified that if $\mu$ is a probability measure on $\mathbb{R}^{3}$ such that

$$
\int_{\mathbb{R}^{3}}|v|^{\alpha} \mu(d v)<+\infty
$$

with the additional assumption that the first-order moments vanish in the case $1<\alpha \leq 2$, then $\widehat{\mu} \in \mathscr{K}^{\alpha}$. The reverse implication, however, is false as it can be seen from the Lévi characteristic function $\widehat{\mu}(\xi)=e^{-|\xi|^{\alpha}}$ with $0<\alpha<2$ which belongs to $\mathscr{K}^{\alpha}$ but

$$
\int_{\mathbb{R}^{3}}|v|^{\alpha} \mu(d v)=+\infty
$$

As a means of treating singularity, we follow Morimoto to consider weak integrability of the kernel $b$ in the form

$$
\int_{0}^{\pi / 2} b(\cos \theta) \sin \theta \sin ^{\alpha_{0}}\left(\frac{\theta}{2}\right) d \theta<+\infty
$$

with $0<\alpha_{0} \leq 2$. It is certainly satisfied by the true Maxwellian kernel $b$ which behaves like (4) as long as $\alpha_{0}>1 / 2$. In addition, we will consider

$$
\begin{aligned}
& \lambda_{\alpha}=\int_{\mathbb{S}^{2}} b\left(\frac{\xi \cdot \sigma}{|\xi|}\right)\left(\frac{\left|\xi^{+}\right|^{\alpha}+\left|\xi^{-}\right|^{\alpha}}{|\xi|^{\alpha}}-1\right) d \sigma \\
&=2 \pi \int_{0}^{\pi / 2} b(\cos \theta) \sin \theta \\
& \quad \times\left[\cos ^{\alpha}\left(\frac{\theta}{2}\right)+\sin ^{\alpha}\left(\frac{\theta}{2}\right)-1\right] d \theta
\end{aligned}
$$

for $0<\alpha \leq 2$, which is independent of $\xi \neq 0$ and finite under condition (20) for all $\alpha_{0} \leq \alpha \leq 2$. Introduced by Cannone and Karch, these quantities will serve as the stability exponents.

To state our results, we set down the precise solution spaces. Let $T>0$ be arbitrary. As it is customary, we denote by $C\left([0, T] ; \mathscr{K}^{\alpha}\right)$ the space of all complex-valued functions $\phi$ on $\mathbb{R}^{3} \times[0, T]$ such that $\phi(\cdot, t) \in \mathscr{K}^{\alpha}\left(\mathbb{R}^{3}\right)$ for each $t \in[0, T]$ and the map $t \mapsto\|\phi(t)-1\|_{\alpha}$ is continuous on $[0, T]$. By the Riemann-Lebesgue lemma, each characteristic function is continuous in $\mathbb{R}^{3}$ and, hence, the space continuity is alluded in the definition of $C\left([0, T] ; \mathscr{K}^{\alpha}\right)$.

In consideration of time regularity, we will write $\Omega^{\alpha}\left(\mathbb{R}^{3} \times\right.$ $[0, T])$ for the space of $\phi \in C\left([0, T] ; \mathscr{K}^{\alpha}\right)$ such that $\phi(\xi, \cdot) \in$ $C([0, T]), \partial_{t} \phi(\xi, \cdot) \in C((0, T))$ for each fixed $\xi \in \mathbb{R}^{3}$. We put

$$
\Omega^{\alpha}\left(\mathbb{R}^{3} \times[0, \infty)\right)=\bigcup_{T>0} \Omega^{\alpha}\left(\mathbb{R}^{3} \times[0, T]\right) .
$$

Our main result for global existence is as follows.

Theorem 1. Assume that the collision kernel b satisfies a weak integrability condition (20) for some $0<\alpha_{0} \leq 2$ and $\alpha_{0} \leq \alpha \leq$ 2. Then, for any initial datum $\phi_{0} \in \mathscr{K}^{\alpha}$, there exists a classical solution $\phi$ to the Cauchy problem (11) in the space $\Omega^{\alpha}\left(\mathbb{R}^{3} \times\right.$ $[0, \infty))$ satisfying

$$
|\phi(\xi, t)| \leq e^{-\nu|\xi|^{2} t} \quad \forall(\xi, t) \in \mathbb{R}^{3} \times[0, \infty) .
$$


A distinctive feature is the existence of a solution satisfying the stated maximum growth estimate which asserts in a sense that the solution stays within Brownian motion for all time.

To state our main result for stability and uniqueness, we put

$$
\begin{aligned}
& \Omega_{\nu}^{\alpha}\left(\mathbb{R}^{3} \times[0, \infty)\right) \\
& =\left\{\phi \in \Omega^{\alpha}\left(\mathbb{R}^{3} \times[0, \infty)\right):|\phi(\xi, t)| \leq e^{-\nu|\xi|^{2} t}\right. \\
& \left.\forall(\xi, t) \in \mathbb{R}^{3} \times[0, \infty)\right\} .
\end{aligned}
$$

Theorem 2. Under the same hypotheses on $\alpha, b$ as in Theorem 1, if $\phi, \psi$ are solutions to Cauchy problem (11) in the space $\Omega_{\nu}^{\alpha}\left(\mathbb{R}^{3} \times[0, \infty)\right)$ corresponding to the initial data $\phi_{0}, \psi_{0} \in \mathscr{K}^{\alpha}$, respectively, then, for all $t \geq 0$,

$$
\begin{aligned}
& \sup _{\xi \in \mathbb{R}^{3}} {\left[e^{\vartheta|\xi|^{2} t} \frac{|\phi(\xi, t)-\psi(\xi, t)|}{|\xi|^{\alpha}}\right] } \\
& \leq e^{\lambda_{\alpha} t} \sup _{\xi \in \mathbb{R}^{3}} \frac{\left|\phi_{0}(\xi)-\psi_{0}(\xi)\right|}{|\xi|^{\alpha}} .
\end{aligned}
$$

In particular, for any initial datum $\phi_{0} \in \mathscr{K}^{\alpha}$, Cauchy problem (11) has at most one solution in the space $\Omega_{\nu}^{\alpha}\left(\mathbb{R}^{3} \times[0, \infty)\right)$.

Upon setting $v=0$, both theorems are reduced to those of Cannone and Karch and Morimoto. In fact, due to a special structure of the Boltzmann-Bobylev operator, to be explained below, the existence theorem is an almost instant consequence of their existence theorem except for some technical points. On the other hand, the stability theorem is not so straightforward and our proof will be carried out along Gronwall-type reasonings.

As some functionals or expressions involving the space and time variables are too lengthy to put effectively, we will often abbreviate the space variables for simplicity in the sequel.

\section{Preliminaries}

A well-known Fourier transform formula states that

$$
e^{-|\xi|^{2} t}=\frac{1}{(4 \pi t)^{3 / 2}} \int_{\mathbb{R}^{3}} e^{-i \xi \cdot v} e^{-|v|^{2} / 4 t} d v \quad\left(\xi \in \mathbb{R}^{3}, t>0\right)
$$

and, hence, it is clear that the Gaussian family $\left\{e^{-|\xi|^{2} t}\right\}_{t \geq 0} \subset$ $\mathscr{K}$ whose probability densities are self-similar Gaussian functions (see, e.g., [21]).

Lemma 3. If $0<\alpha \leq 2$ and $t>0$, then

$$
\sup _{\xi \in \mathbb{R}^{3}} \frac{1-e^{-|\xi|^{2} t}}{|\xi|^{\alpha}} \leq t^{\alpha / 2}
$$

Proof. Observe that

$$
\begin{array}{r}
\sup _{\xi \in \mathbb{R}^{3}} \frac{1-e^{-|\xi|^{2} t}}{|\xi|^{\alpha}}=t^{\alpha / 2} \cdot \sup _{r>0} g_{\alpha}(r) \\
\text { with } g_{\alpha}(r)=\left(\frac{1-e^{-r}}{r^{\alpha / 2}}\right) .
\end{array}
$$

Since $g_{\alpha}$ is a smooth function on $(0, \infty)$ with

$$
\begin{gathered}
g_{\alpha}(r) \leq \min \left(r^{1-\alpha / 2}, 1\right), \\
\lim _{r \rightarrow 0+} g_{\alpha}(r)=\lim _{r \rightarrow \infty} g_{\alpha}(r)=0,
\end{gathered}
$$

the assertion follows.

The Boltzmann-Bobylev operator defined in (10) takes the form

$$
\mathscr{B}(\phi)(\xi)=\int_{\mathbb{S}^{2}} b\left(\frac{\xi \cdot \sigma}{|\xi|}\right)\left[\phi\left(\xi^{+}\right) \phi\left(\xi^{-}\right)-\phi(\xi)\right] d \sigma
$$

for each characteristic function $\phi$. We set $\mathscr{B}(\phi)(0)=0$ in the sequel.

For a nonzero $\xi \in \mathbb{R}^{3}$, by considering a parametrization of the unit sphere in terms of the deviation angle from $\xi /|\xi|$, it is well known that

$$
\begin{aligned}
\mathscr{B}(\phi)(\xi)=\int_{0}^{\pi / 2} b(\cos \theta) \sin \theta \\
\times\left\{\int_{\mathbb{S}^{1}(\xi)}\left[\phi\left(\xi^{+}\right) \phi\left(\xi^{-}\right)-\phi(\xi)\right] d \omega\right\} d \theta
\end{aligned}
$$

in which $\mathbb{S}^{1}(\xi)=\mathbb{S}^{2} \cap \xi^{\perp}$ and $d \omega$ denotes the area measure on the unit circle $\mathbb{S}^{1} \subset \mathbb{R}^{3}$. As it is defined in (9), the spherical variables $\xi^{+}, \xi^{-}$are expressed in terms of $\theta, \omega$ via

$$
\sigma=\cos \theta \frac{\xi}{|\xi|}+\sin \theta \omega
$$

The following are due to Morimoto [20, page 555]. We put

$$
\mu_{\alpha}=2 \pi \int_{0}^{\pi / 2} b(\cos \theta) \sin \theta \sin ^{\alpha}\left(\frac{\theta}{2}\right) d \theta,
$$

which is finite under condition (20) for any $\alpha_{0} \leq \alpha \leq 2$.

Lemma 4. For $0<\alpha \leq 2$, assume that the kernel $b$ satisfies the condition $\mu_{\alpha}<+\infty$. Let $\phi \in \mathscr{K}^{\alpha}$ and $\xi \in \mathbb{R}^{3}-\{0\}$. Then,

$$
\begin{aligned}
& \left|\int_{\mathbb{S}^{1}(\xi)}\left[\phi\left(\xi^{+}\right) \phi\left(\xi^{-}\right)-\phi(\xi)\right] d \omega\right| \\
& \quad \leq 16 \pi\|\phi-1\|_{\alpha}|\xi|^{\alpha} \sin ^{\alpha}\left(\frac{\theta}{2}\right)
\end{aligned}
$$

for each $\theta \in(0, \pi / 2]$. Moreover,

$$
|\mathscr{B}(\phi)(\xi)| \leq 8 \mu_{\alpha}\|\phi-1\|_{\alpha}|\xi|^{\alpha} .
$$


As an application, we have the following time-continuity property.

Lemma 5. For $0<\alpha \leq 2$, assume that the kernel $b$ satisfies the condition $\mu_{\alpha}<+\infty$ and $T>0$. If $\phi \in C\left([0, T] ; \mathscr{K}^{\alpha}\right)$ and $\phi(\xi, \cdot) \in C([0, T])$ for each $\xi \in \mathbb{R}^{3}$, then $\mathscr{B}(\phi)(\xi, \cdot) \in C([0, T])$ for each $\xi \in \mathbb{R}^{3}$.

Proof. Fix a nonzero $\xi \in \mathbb{R}^{3}$ and $t_{0} \in[0, T]$. For any sequence $\left(t_{n}\right) \subset[0, T]$ with $t_{n} \rightarrow t_{0}$, we may write, with the aid of (31),

$$
\begin{aligned}
& \mathscr{B}(\phi)\left(\xi, t_{n}\right) \\
& =\int_{\mathbb{S}^{2}} b\left(\frac{\xi \cdot \sigma}{|\xi|}\right)\left[\phi\left(\xi^{+}, t_{n}\right) \phi\left(\xi^{-}, t_{n}\right)-\phi\left(\xi, t_{n}\right)\right] d \sigma \\
& =\int_{0}^{\pi / 2} b(\cos \theta) \sin \theta A_{n}(\xi, \theta) d \theta \quad \text { where } \\
& A_{n}(\xi, \theta)=\int_{\mathbb{S}^{1}(\xi)}\left[\phi\left(\xi^{+}, t_{n}\right) \phi\left(\xi^{-}, t_{n}\right)-\phi\left(\xi, t_{n}\right)\right] d \omega .
\end{aligned}
$$

By the estimate (34), we notice that

$$
\begin{aligned}
& \left|A_{n}(\xi, \theta)\right| \leq 16 \pi\left\|\phi\left(t_{n}\right)-1\right\|_{\alpha}|\xi|^{\alpha} \sin ^{\alpha}\left(\frac{\theta}{2}\right) \\
& \quad \leq 16 \pi C_{\alpha}(T)|\xi|^{\alpha} \sin ^{\alpha}\left(\frac{\theta}{2}\right) \quad \text { where } \\
& C_{\alpha}(T)=\max _{t \in[0, T]}\|\phi(t)-1\|_{\alpha} .
\end{aligned}
$$

By the continuity of $t \mapsto\|\phi(t)-1\|_{\alpha}$, we have $C_{\alpha}(T)<+\infty$. Since

$$
\begin{aligned}
& b(\cos \theta) \sin \theta\left|A_{n}(\xi, \theta)\right| \\
& \quad \leq 16 \pi C_{\alpha}(T)|\xi|^{\alpha} b(\cos \theta) \sin \theta \sin ^{\alpha}\left(\frac{\theta}{2}\right) \\
& \quad \equiv A(\xi, \theta)
\end{aligned}
$$

uniformly in $n$ and the definition of $\mu_{\alpha}$ gives

$$
\int_{0}^{\pi / 2} A(\xi, \theta) d \theta=8 \mu_{\alpha} C_{\alpha}(T)|\xi|^{\alpha}<+\infty,
$$

we may apply Lebesgue's dominated convergence theorem to evaluate the limit under the integral sign $\lim _{n \rightarrow \infty} \mathscr{B}(\phi)\left(\xi, t_{n}\right)=\mathscr{B}(\phi)\left(\xi, t_{0}\right)$, which proves continuity at $t_{0}$.

\section{Global Existence}

An important feature of the Boltzmann-Bobylev operator $\mathscr{B}$ is that it satisfies the pointwise identity

$$
e^{h(t)|\xi|^{2}} \mathscr{B}(\phi)(\xi, t)=\mathscr{B}\left(e^{h(t)|\xi|^{2}} \phi\right)(\xi, t)
$$

for any scalar-valued function $h$ defined on $[0, \infty)$ and for any scalar-valued function $\phi$ on $\mathbb{R}^{3} \times[0, \infty)$, which results from $\left|\xi^{+}\right|^{2}+\left|\xi^{-}\right|^{2}=|\xi|^{2}$ for all $\xi \in \mathbb{R}^{3}$ and $\sigma \in \mathbb{S}^{2}$. As a consequence, at the formal level, it is straightforward to find that if $\phi$ is a solution to Cauchy problem (13) of the Fouriertransformed Boltzmann equation, then $e^{-v|\xi|^{2} t} \phi$ is a solution to Cauchy problem (11) of our consideration.

To be rigorous, we begin with quoting the existence theorem of Cannone and Karch [19] and Morimoto [20] in a combined manner.

Theorem 6. Assume that $b$ satisfies (20) for some $0<\alpha_{0} \leq 2$. Let $\alpha_{0} \leq \alpha \leq 2$ and $\phi_{0} \in \mathscr{K}^{\alpha}$. Then, there exists a unique classical solution $\phi$ to Cauchy problem (13) in the space $\Omega^{\alpha}\left(\mathbb{R}^{3} \times\right.$ $[0, \infty))$.

Remark 7. In their work, Cannone and Karch constructed a unique solution on the space $C\left([0, \infty) ; \mathscr{K}^{\alpha}\right)$ without mentioning time-regularity conditions. Since $\mathscr{K}^{\alpha}$ is not a Banach space, the meaning of a classical solution to Cauchy problem (20) is not so clear in this space. Inspecting their proof and making use of the time continuity of the Boltzmann-Bobylev operator as stated in Lemma 5, however, it is not hard to find that their solution is indeed a classical solution in the space $\Omega^{\alpha}\left(\mathbb{R}^{3} \times[0, \infty)\right)$ for which the partial derivative in time is taken in the usual pointwise sense.

Let us consider an equivalent formulation of (13):

$$
\phi(\xi, t)=\phi_{0}(\xi)+\int_{0}^{t} \mathscr{B}(\phi)(\xi, \tau) d \tau,
$$

where the time integration is taken in the usual Riemann sense. By Lemma 5 , if $\phi \in C\left([0, T] ; \mathscr{K}^{\alpha}\right)$ and $\phi(\xi, t)$ is continuous in $t$ for each fixed $\xi$, then this integral is well defined for a kernel $b$ satisfying $\mu_{\alpha}<+\infty$.

We will need a technical lemma in support of Theorem 6.

Lemma 8. For $0<\alpha \leq 2$, let $\mu_{\alpha}<+\infty$ and $\phi_{0} \in \mathscr{K}^{\alpha}$. Assume that $\phi \in C\left([0, T] ; \mathscr{K}^{\alpha}\right)$ and $\phi(\xi, t)$ is continuous in $t \in[0, T]$ for each fixed $\xi$. If $\phi$ is a solution to (41), then, for all $s, t \in[0, T]$,

(i) $\|\phi(t)-1\|_{\alpha} \leq e^{8 \mu_{\alpha} t}\left\|\phi_{0}-1\right\|_{\alpha}$,

(ii) $\|\phi(t)-\phi(s)\|_{\alpha} \leq\left(8 \mu_{\alpha} e^{8 \mu_{\alpha} T}\left\|\phi_{0}-1\right\|_{\alpha}\right)|t-s|$.

Proof. (i) An application of Lemma 4 yields

$$
\|\phi(t)-1\|_{\alpha} \leq\left\|\phi_{0}-1\right\|_{\alpha}+8 \mu_{\alpha} \int_{0}^{t}\|\phi(\tau)-1\|_{\alpha} d \tau,
$$

which yields the desired estimate in view of Gronwall's lemma.

(ii) Assuming $s<t$, we apply Lemma 4 once again to find

$$
\begin{aligned}
\| \phi(t) & -\phi(s) \|_{\alpha} \\
& \leq \int_{s}^{t} \sup _{\xi \in \mathbb{R}^{3}} \frac{|\mathscr{B}(\phi)(\xi, \tau)|}{|\xi|^{\alpha}} d \tau \\
& \leq 8 \mu_{\alpha}\left(\max _{\tau \in[0, T]}\|\phi(\tau)-1\|_{\alpha}\right)|t-s|,
\end{aligned}
$$

which yields the desired estimate upon combining with (i). 
Proof of Theorem 1. Since the stated assumptions on $b$ and $\phi_{0}$ are the same as those of Theorem 6 , there exists a unique solution $\phi$ to Cauchy problem (13) in the space $\Omega^{\alpha}\left(\mathbb{R}^{3} \times\right.$ $[0, \infty))$. Put

$$
\Phi(\xi, t)=e^{-v|\xi|^{2} t} \phi(\xi, t) .
$$

We will verify that $\Phi$ is a solution to Cauchy problem (11) satisfying the stated maximum growth estimate.

(i) Clearly $\Phi(\cdot, t) \in \mathscr{K}$ for any fixed $t \geq 0$. Moreover, Lemma 3 gives

$$
\begin{aligned}
& \frac{|\Phi(\xi, t)-1|}{|\xi|^{\alpha}} \\
& \quad \leq e^{-v|\xi|^{2} t} \frac{|\phi(\xi, t)-1|}{|\xi|^{\alpha}}+\frac{1-e^{-v|\xi|^{2} t}}{|\xi|^{\alpha}} \\
& \quad \leq\|\phi(t)-1\|_{\alpha}+(\nu t)^{\alpha / 2},
\end{aligned}
$$

which implies $\Phi(\cdot, t) \in \mathscr{K}^{\alpha}$ for any fixed $t \geq 0$ with

$$
\|\Phi(t)-1\|_{\alpha} \leq e^{8 \mu_{\alpha} t}\left\|\phi_{0}-1\right\|_{\alpha}+(\nu t)^{\alpha / 2} .
$$

(ii) For $s, t \in[0, T]$, with an arbitrary $T>0$, writing

$$
\begin{aligned}
\Phi(t)-\Phi(s)= & -e^{-\nu|\xi|^{2} s}\left[1-e^{-v|\xi|^{2}(t-s)}\right] \phi(t) \\
& +e^{-\nu|\xi|^{2} s}[\phi(t)-\phi(s)],
\end{aligned}
$$

we deduce from Lemmas 3 and 8

$$
\begin{aligned}
& \|\Phi(t)-\Phi(s)\|_{\alpha} \\
& \leq(\nu|t-s|)^{\alpha / 2}+\|\phi(t)-\phi(s)\|_{\alpha} \\
& \quad \leq C_{T}|t-s|^{\alpha / 2} \text { where } \\
& C_{T}=\nu^{\alpha / 2}+8 \mu_{\alpha} e^{8 \mu_{\alpha} T}\left\|\phi_{0}-1\right\|_{\alpha} T^{1-\alpha / 2} .
\end{aligned}
$$

Thus, the map $t \mapsto\|\Phi(t)-1\|_{\alpha}$ is Lipschitz continuous in $[0, T]$ for

$$
\left|\|\Phi(t)-1\|_{\alpha}-\|\Phi(s)-1\|_{\alpha}\right| \leq\|\Phi(t)-\Phi(s)\|_{\alpha} .
$$

Therefore, $\Phi \in \Omega^{\alpha}\left(\mathbb{R}^{3} \times[0, \infty)\right)$ for the time-regularity conditions are obviously valid. In particular, Lemmas 4 and 5 imply that $\mathscr{B}(\Phi)$ is well defined with

$$
|\mathscr{B}(\Phi)(\xi, t)| \leq 8 \mu_{\alpha}\|\Phi(t)-1\|_{\alpha}|\xi|^{\alpha}
$$

for each $(\xi, t) \in \mathbb{R}^{3} \times[0, \infty)$ and $\mathscr{B}(\Phi)(\xi, t)$ is continuous in $t$ for each $\xi$. Clearly, $\Phi(\xi, 0)=\phi_{0}(\xi)$. We calculate

$$
\begin{aligned}
\partial_{t} \Phi(\xi, t) & \\
& =-\nu|\xi|^{2} e^{-v|\xi|^{2} t} \phi(\xi, t)+e^{-v|\xi|^{2} t} \partial_{t} \phi(\xi, t) \\
& =-v|\xi|^{2} \Phi(\xi, t)+e^{-v|\xi|^{2} t} \mathscr{B}(\phi)(\xi, t) \\
& =-\nu|\xi|^{2} \Phi(\xi, t)+\mathscr{B}(\Phi)(\xi, t)
\end{aligned}
$$

for all $(\xi, t) \in \mathbb{R}^{3} \times(0, \infty)$, where we have used (40). Thus, $\Phi$ satisfies Cauchy problem (11). Since it is obvious that

$$
|\Phi(\xi, t)| \leq e^{-v|\xi|^{2} t} \quad \forall(\xi, t) \in \mathbb{R}^{3} \times[0, \infty),
$$

our proof of Theorem 1 is complete.

Remark 9. In our forthcoming paper [22], we study the Cauchy problem for the Boltzmann equation coupled with fractional Laplacian diffusion terms on the Fourier transform side in which we give direct proofs of global existence.

\section{Uniqueness and Stability of Solutions}

To proceed our proof for stability of solutions, we begin with estimating the time-growth behavior of $\|\phi(t)-1\|_{\alpha}$ for each solution $\phi$ of Cauchy problem (11) or integral equation (12).

Lemma 10. Under the same hypotheses on $\alpha, b, \phi$ as stated in Lemma 8, if $\phi$ is a solution to (12), then

$$
\begin{array}{r}
\|\phi(t)-1\|_{\alpha} \leq e^{8 \mu_{\alpha} t}\left[\left\|\phi_{0}-1\right\|_{\alpha}+(\nu t)^{\alpha / 2}\right] \\
(t \geq 0) .
\end{array}
$$

Proof. Writing

$$
\begin{aligned}
\frac{\phi(\xi, t)-1}{|\xi|^{\alpha}}= & \frac{e^{-v|\xi|^{2} t} \phi_{0}(\xi)-1}{|\xi|^{\alpha}} \\
& +\int_{0}^{t} e^{-\nu|\xi|^{2}(t-\tau)} \frac{\mathscr{B}(\phi)(\xi, \tau)}{|\xi|^{\alpha}} d \tau
\end{aligned}
$$

and applying Lemma 4, it is straightforward to obtain

$$
\begin{aligned}
\|\phi(t)-1\|_{\alpha} \leq & \left\|\phi_{0}-1\right\|_{\alpha}+(v t)^{\alpha / 2} \\
& +8 \mu_{\alpha} \int_{0}^{t}\|\phi(\tau)-1\|_{\alpha} d \tau .
\end{aligned}
$$

A Gronwall-type argument yields

$$
\begin{aligned}
\| \phi(t) & -1 \|_{\alpha} \\
& \leq e^{8 \mu_{\alpha} t}\left\{\left\|\phi_{0}-1\right\|_{\alpha}+\frac{\alpha \nu^{\alpha / 2}}{2} \int_{0}^{t} e^{-8 \mu_{\alpha} \tau} \tau^{\alpha / 2-1} d \tau\right\} \\
& \leq e^{8 \mu_{\alpha} t}\left[\left\|\phi_{0}-1\right\|_{\alpha}+(\nu t)^{\alpha / 2}\right] .
\end{aligned}
$$

Proof of Theorem 2. We will prove the stated stability inequality for each $t \in[0, T]$ with an arbitrarily fixed $T>0$.

Let us consider a monotone sequence $\left(b_{n}\right)$ of kernels obtained from $b$ by cutting off the singularity at $\theta=0$ in the manner

$$
b_{n}(\cos \theta)=b(\cos \theta) \chi_{[1 / n, \pi / 2]}(\theta), \quad n=1,2, \ldots
$$

Since $b$ is assumed to be at least bounded away from $\theta=0$, it is clear that each $b_{n}$ is integrable on the unit sphere, $b_{n} \leq b$ 
and $b_{n} \rightarrow b$ monotonically. Setting $b_{n}^{r}=b-b_{n}$ for each $n$, we introduce two sequences of operators $\left(\mathscr{G}_{n}\right),\left(\mathscr{R}_{n}\right)$ defined by

$$
\begin{gathered}
\mathscr{G}_{n}(\phi)(\xi)=\int_{\mathbb{S}^{2}} b_{n}\left(\frac{\xi \cdot \sigma}{|\xi|}\right) \phi\left(\xi^{+}\right) \phi\left(\xi^{-}\right) d \sigma \\
\mathscr{R}_{n}(\phi)(\xi)=\int_{\mathbb{S}^{2}} b_{n}^{r}\left(\frac{\xi \cdot \sigma}{|\xi|}\right)\left[\phi\left(\xi^{+}\right) \phi\left(\xi^{-}\right)-\phi(\xi)\right] d \sigma .
\end{gathered}
$$

Suppose that $\phi, \psi \in \Omega_{\nu}^{\alpha}\left(\mathbb{R}^{3} \times[0, \infty)\right)$ are solutions to Cauchy problem (11) with the initial data $\phi_{0}, \psi_{0} \in \mathscr{K}^{\alpha}$, respectively. Then,

$$
\begin{aligned}
\partial_{t}(\phi & -\psi)+\left(\left\|b_{n}\right\|_{1}+\nu|\xi|^{2}\right)(\phi-\psi) \\
& =\left[\mathscr{G}_{n}(\phi)-\mathscr{G}_{n}(\psi)\right]+\left[\mathscr{R}_{n}(\phi)-\mathscr{R}_{n}(\psi)\right]
\end{aligned}
$$

for which we denote

$$
\left\|b_{n}\right\|_{1}=2 \pi \int_{1 / n}^{\pi / 2} b(\cos \theta) \sin \theta d \theta<+\infty .
$$

Upon setting

$$
U(\xi, t)=e^{\nu|\xi|^{2} t}\left[\frac{\phi(\xi, t)-\psi(\xi, t)}{|\xi|^{\alpha}}\right]
$$

for $\xi \neq 0$ and $U(0, t)=0$, the above identity implies

$$
\begin{aligned}
& \left|\partial_{t}\left[e^{\left\|b_{n}\right\|_{1} t} U(\xi, t)\right]\right| \\
& \leq e^{\left(\left\|b_{n}\right\|_{1}+v|\xi|^{2}\right) t} \\
& \quad \times\left\{\left|\frac{\mathscr{G}_{n}(\phi)-\mathscr{G}_{n}(\psi)}{|\xi|^{\alpha}}\right|+\left|\frac{\mathscr{R}_{n}(\phi)-\mathscr{R}_{n}(\psi)}{|\xi|^{\alpha}}\right|\right\} .
\end{aligned}
$$

Let $\rho>0$ be arbitrary. Put $U_{\rho}(t)=\sup _{|\xi| \leq \rho}|U(\xi, t)|$ and

$$
\begin{aligned}
\gamma_{n, \alpha} & =\int_{\mathbb{S}^{2}} b_{n}\left(\frac{\xi \cdot \sigma}{|\xi|}\right)\left(\frac{\left|\xi^{+}\right|^{\alpha}+\left|\xi^{-}\right|^{\alpha}}{|\xi|^{\alpha}}\right) d \sigma \\
& =2 \pi \int_{1 / n}^{\pi / 2} b(\cos \theta) \sin \theta\left[\cos ^{\alpha}\left(\frac{\theta}{2}\right)+\sin ^{\alpha}\left(\frac{\theta}{2}\right)\right] d \theta .
\end{aligned}
$$

For $|\xi| \leq \rho$, we make use of $\left|\xi^{+}\right| \leq|\xi|$ to estimate

$$
\begin{aligned}
\mid \phi\left(\xi^{+}, t\right) & -\psi\left(\xi^{+}, t\right) \mid \\
\leq & e^{-\gamma\left|\xi^{+}\right|^{2} t}\left|\xi^{+}\right|^{\alpha} \\
& \times\left\{e^{\nu\left|\xi^{+}\right|^{2} t}\left|\frac{\phi\left(\xi^{+}, t\right)-\psi\left(\xi^{+}, t\right)}{\left|\xi^{+}\right|^{\alpha}}\right|\right\} \\
\leq & e^{-\nu\left|\xi^{+}\right|^{2} t}\left|\xi^{+}\right|^{\alpha} U_{\rho}(t) .
\end{aligned}
$$

Likewise, we make use of $\left|\xi^{-}\right| \leq|\xi|$ to estimate

$$
\left|\phi\left(\xi^{-}, t\right)-\psi\left(\xi^{-}, t\right)\right| \leq e^{-\gamma\left|\xi^{-}\right|^{2} t}\left|\xi^{-}\right|^{\alpha} U_{\rho}(t)
$$

for all $|\xi| \leq \rho$. Since $|\phi(\xi, t)| \leq e^{-\nu|\xi|^{2} t},|\psi(\xi, t)| \leq e^{-\nu|\xi|^{2} t}$, we find

$$
\begin{gathered}
\left|\phi\left(\xi^{+}, t\right) \phi\left(\xi^{-}, t\right)-\psi\left(\xi^{+}, t\right) \psi\left(\xi^{-}, t\right)\right| \\
\leq e^{-v|\xi|^{2} t}\left(\left|\xi^{+}\right|^{\alpha}+\left|\xi^{-}\right|^{\alpha}\right) U_{\rho}(t)
\end{gathered}
$$

for all $|\xi| \leq \rho$. Henceforth, it is straightforward to deduce

$$
e^{\nu|\xi|^{2} t}\left|\frac{\mathscr{G}_{n}(\phi)-\mathscr{G}_{n}(\psi)}{|\xi|^{\alpha}}\right| \leq \gamma_{n, \alpha} U_{\rho}(t)
$$

for all $|\xi| \leq \rho$. On the other hand, Lemma 4 gives

$$
\begin{aligned}
\left|\mathscr{R}_{n}(\phi)(\xi, t)\right|= & 16 \pi\|\phi(t)-1\|_{\alpha}|\xi|^{\alpha} \\
& \times \int_{0}^{1 / n} b(\cos \theta) \sin \theta \sin ^{\alpha}\left(\frac{\theta}{2}\right) d \theta .
\end{aligned}
$$

By considering similar estimate for $\mathscr{R}_{n}(\psi)$, hence, we note

$$
e^{\nu|\xi|^{2} t}\left|\frac{\mathscr{R}_{n}(\phi)-\mathscr{R}_{n}(\psi)}{|\xi|^{\alpha}}\right| \leq M_{n}
$$

for all $|\xi| \leq \rho$ and $t \in[0, T]$, where we put

$$
\begin{aligned}
& M_{n}=M(\rho, T) \int_{0}^{1 / n} b(\cos \theta) \sin \theta \sin ^{\alpha}\left(\frac{\theta}{2}\right) d \theta, \\
& M(\rho, T)= 16 \pi e^{\nu \rho^{2} T} \\
& \times \max _{t \in[0, T]}\left(\|\phi(t)-1\|_{\alpha}+\|\psi(t)-1\|_{\alpha}\right) .
\end{aligned}
$$

In view of the growth estimate of Lemma 10,

$$
\begin{aligned}
& M(\rho, T) \\
& \leq 16 \pi e^{\left(\nu \rho^{2}+8 \mu_{\alpha}\right) T} \\
& \quad \times\left[\left\|\phi_{0}-1\right\|_{\alpha}+\left\|\psi_{0}-1\right\|_{\alpha}+2(\nu T)^{\alpha / 2}\right]<+\infty,
\end{aligned}
$$

and so an application of Lebesgue's dominated convergence theorem shows $M_{n} \rightarrow 0$ as $n \rightarrow \infty$ under the assumption $\mu_{\alpha}<+\infty$.

Now, estimates (68) and (70) imply

$$
\left|\partial_{t}\left[e^{\left\|b_{n}\right\|_{1} t} U(\xi, t)\right]\right| \leq \gamma_{n, \alpha} e^{\left\|b_{n}\right\|_{1} t} U_{\rho}(t)+M_{n} e^{\left\|b_{n}\right\|_{1} t}
$$

for all $|\xi| \leq \rho, t \in[0, T]$. A standard Gronwall-type argument gives

$$
U_{\rho}(t) \leq e^{\left(\gamma_{n, \alpha}-\left\|b_{n}\right\|_{1}\right) t} U_{\rho}(0)+\frac{M_{n}}{\gamma_{n, \alpha}-\left\|b_{n}\right\|_{1}}\left[e^{\left(\gamma_{n, \alpha}-\left\|b_{n}\right\|_{1}\right) t}-1\right] .
$$


Since

$$
\begin{aligned}
\gamma_{n, \alpha}-\left\|b_{n}\right\|_{1}=2 \pi \int_{1 / n}^{\pi / 2} b(\cos \theta) \sin \theta \\
\quad \times\left[\cos ^{\alpha}\left(\frac{\theta}{2}\right)+\sin ^{\alpha}\left(\frac{\theta}{2}\right)-1\right] d \theta,
\end{aligned}
$$

we notice $0<\gamma_{n, \alpha}-\left\|b_{n}\right\|_{1} \rightarrow \lambda_{\alpha}$ increasingly as $n \rightarrow \infty$. Passing to the limit, we conclude $U_{\rho}(t) \leq e^{\lambda_{\alpha} t} U_{\rho}(0)$ for all $t \in[0, T]$. Letting $\rho \rightarrow+\infty$, we finally obtain

$$
\sup _{\xi \in \mathbb{R}^{3}}|U(\xi, t)| \leq e^{\lambda_{\alpha} t} \sup _{\xi \in \mathbb{R}^{3}}|U(\xi, 0)|,
$$

which is equivalent to the desired stability estimate on $[0, T]$.

\section{Concluding Remarks}

Having established global existence and uniqueness and stability of solutions to the Fourier-transformed version of Fokker-Planck-Boltzmann equation on the space $\mathscr{K}^{\alpha}$, we end our paper with a few additional remarks.

(a) Concerning Theorem 1, while it asserts that there exists a solution $\phi$ of Cauchy problem (11) satisfying

$$
|\phi(\xi, t)| \leq e^{-\nu|\xi|^{2} t} \quad \forall(\xi, t) \in \mathbb{R}^{3} \times[0, \infty),
$$

a natural question is whether this dominating property would hold for any solution of (11). The answer is affirmative in the case when the collision kernel $b$ satisfies Grad's angular cutoff assumption.

Suppose $b \in L^{1}\left(\mathbb{S}^{2}\right)$ and $\phi_{0} \in \mathscr{K}$. If $\phi$ is a solution to (11) in the space $C([0, \infty) ; \mathscr{K})$, then necessarily (77) holds.

As it can be proved in an elementary way, we leave its verification to the interested reader. For the singular case of $b$, however, we were not able to draw any conclusion.

(b) In the cutoff case of $b$, it is possible to construct an explicit solution of Cauchy problem (11) by using the Wild sum method as developed in [15, 17, 23]. Assuming $\|b\|_{L^{1}\left(\mathbb{S}^{2}\right)}=1$, if we follow the same known method, then it is straightforward to derive

$$
\phi(\xi, t)=e^{-\left(1+\nu|\xi|^{2}\right) t} \sum_{n=0}^{\infty} u_{n}(\xi)\left(1-e^{-t}\right)^{n},
$$

where $u_{0}=\phi_{0}$ is the initial datum and

$$
u_{n+1}(\xi)=\frac{1}{n+1} \sum_{j=0}^{n} \int_{\mathbb{S}^{2}} b\left(\frac{\xi \cdot \sigma}{|\xi|}\right) u_{j}\left(\xi^{+}\right) u_{n-j}\left(\xi^{-}\right) d \sigma
$$

for $n=0,1, \ldots$. It can be shown plainly that if $\phi_{0} \in$ $\mathscr{K}^{\alpha}$, then this explicit solution $\phi \in \mathscr{K}^{\alpha}$ for $0<$ $\alpha \leq 2$. By uniqueness, this solution coincides with the solution of Theorem 1 . (c) Concerning the asymptotic behavior of a solution $\phi$ to Cauchy problem (11), an important question common in kinetic theory is whether there exists a steady-state equilibrium $\phi_{\infty}$ such that $\phi \rightarrow \phi_{\infty}$ as $t \rightarrow \infty$ in some sense. For instance, in the inelastic case, it is shown that there exists such steady-state equilibrium for a solution of the Cauchy problem for the corresponding Fokker-Planck-Boltzmann equation (see [17] and further references therein). In the elastic case, however, it is likely that the answer would be negative in view of the pointwise behavior $\phi(\xi, t) \rightarrow$ 0 due to growth estimate (77). A seemingly reasonable alternative is to investigate if the solution gets close to the Gaussian $e^{-v|\xi|^{2} t}$ in an appropriate sense.

\section{Conflict of Interests}

The authors declare that there is no conflict of interests regarding the publication of this paper.

\section{Acknowledgments}

Yong-Kum Cho is supported by National Research Foundation of Korea Grant funded by the Korean Government (no. 20130301) and 2013-14 Chung-Ang University Sabbatical Research Grant.

\section{References}

[1] C. Villani, "A review of mathematical topics in collisional kinetic theory," in Handbook of Mathematical Fluid Dynamics, vol. 1, pp. 71-305, North-Holland, Amsterdam, The Netherlands, 2002.

[2] K. Hamdache, "Estimations uniformes des solutions de l'equation de Boltzmann par les methodes de viscosité artificielle et de diffusion de Fokker-Planck," Comptes Rendus de l'Académie des Sciences, vol. 302, no. 5, pp. 187-190, 1986.

[3] R. J. DiPerna and P.-L. Lions, "On the Fokker-PlanckBoltzmann equation," Communications in Mathematical Physics, vol. 120, no. 1, pp. 1-23, 1988.

[4] H.-L. Li and A. Matsumura, "Behaviour of the Fokker-PlanckBoltzmann equation near a Maxwellian," Archive for Rational Mechanics and Analysis, vol. 189, no. 1, pp. 1-44, 2008.

[5] L. Xiong, T. Wang, and L. Wang, "Global existence and decay of solutions to the Fokker-Planck-Boltzmann equation," Kinetic and Related Models, vol. 7, no. 1, pp. 169-194, 2014.

[6] M.-Y. Zhong and H.-L. Li, "Long time behavior of the FokkerPlanck-Boltzmann equation with soft potential," Quarterly of Applied Mathematics, vol. 70, no. 4, pp. 721-742, 2012.

[7] H.-L. Li, "Diffusive property of the Fokker-Planck-Boltzmann equation," Bulletin of the Institute of Mathematics, vol. 2, no. 4, pp. 921-933, 2007.

[8] T. Goudon, "On Boltzmann equations and Fokker-Planck asymptotics: influence of grazing collisions," Journal of Statistical Physics, vol. 89, no. 3-4, pp. 751-776, 1997.

[9] L. Arkeryd, "On the Boltzmann equation. I. Existence," Archive for Rational Mechanics and Analysis, vol. 45, pp. 1-16, 1972.

[10] L. Arkeryd, "Intermolecular forces of infinite range and the Boltzmann equation," Archive for Rational Mechanics and Analysis, vol. 77, no. 1, pp. 11-21, 1981. 
[11] C. Villani, "On a new class of weak solutions to the spatially homogeneous Boltzmann and Landau equations," Archive for Rational Mechanics and Analysis, vol. 143, no. 3, pp. 273-307, 1998.

[12] A. V. Bobylev, "Fourier transform method in the theory of the Boltzmann equation for Maxwell molecules," Doklady Akademii Nauk SSSR, vol. 225, pp. 1041-1044, 1975.

[13] R. M. Blumenthal and R. K. Getoor, "Some theorems on stable processes," Transactions of the American Mathematical Society, vol. 95, pp. 263-273, 1960.

[14] M. Bisi, J. A. Carrillo, and G. Toscani, "Contractive metrics for a Boltzmann equation for granular gases: diffusive equilibria," Journal of Statistical Physics, vol. 118, no. 1-2, pp. 301-331, 2005.

[15] A. Pulvirenti and G. Toscani, "The theory of the nonlinear Boltzmann equation for Maxwell molecules in Fourier representation," Annali di Matematica Pura ed Applicata, vol. 171, pp. 181-204, 1996.

[16] G. Toscani and C. Villani, "Probability metrics and uniqueness of the solution to the Boltzmann equation for a Maxwell gas," Journal of Statistical Physics, vol. 94, no. 3-4, pp. 619-637, 1999.

[17] J. A. Carrillo and G. Toscani, "Contractive probability metrics and asymptotic behavior of dissipative kinetic equations," Rivista di Matematica della Università di Parma, vol. 6, pp. 75-198, 2007.

[18] A. V. Bobylev and C. Cercignani, "Self-similar solutions of the Boltzmann equation and their applications," Journal of Statistical Physics, vol. 106, no. 5-6, pp. 1039-1071, 2002.

[19] M. Cannone and G. Karch, "Infinite energy solutions to the homogeneous Boltzmann equation," Communications on Pure and Applied Mathematics, vol. 63, no. 6, pp. 747-778, 2010.

[20] Y. Morimoto, "A remark on Cannone-Karch solutions to the homogeneous Boltzmann equation for Maxwellian molecules," Kinetic and Related Models, vol. 5, no. 3, pp. 551-561, 2012.

[21] B. Petersen, Introduction to the Fourier Transform \& PseudoDifferential Operators, Pitman, 1983.

[22] Y.-K. Cho, "On the Boltzmann equation with the symmetric stable Lévi process," To appear in Kinetic and Related Models.

[23] E. Wild, "On Boltzmann's equation in the kinetic theory of gases," Mathematical Proceedings of the Cambridge Philosophical Society, vol. 47, pp. 602-609, 1951. 


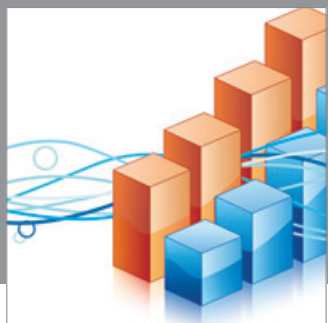

Advances in

Operations Research

mansans

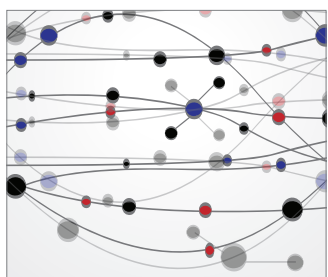

The Scientific World Journal
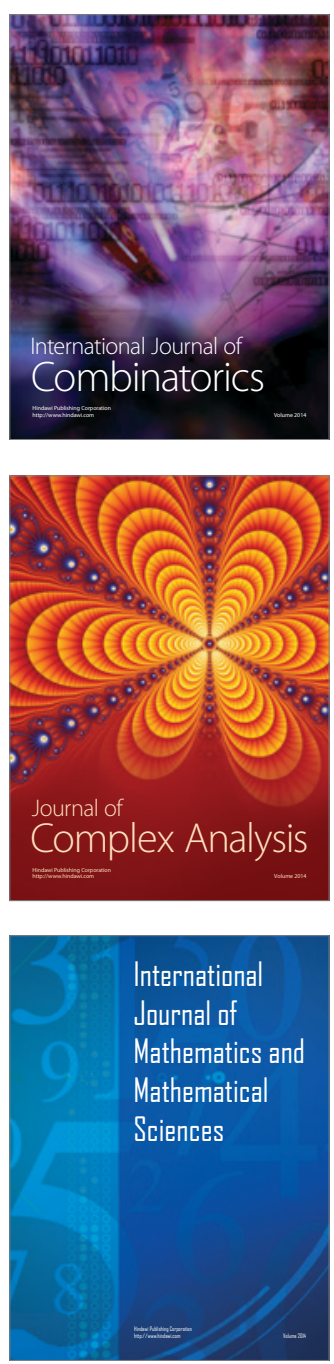
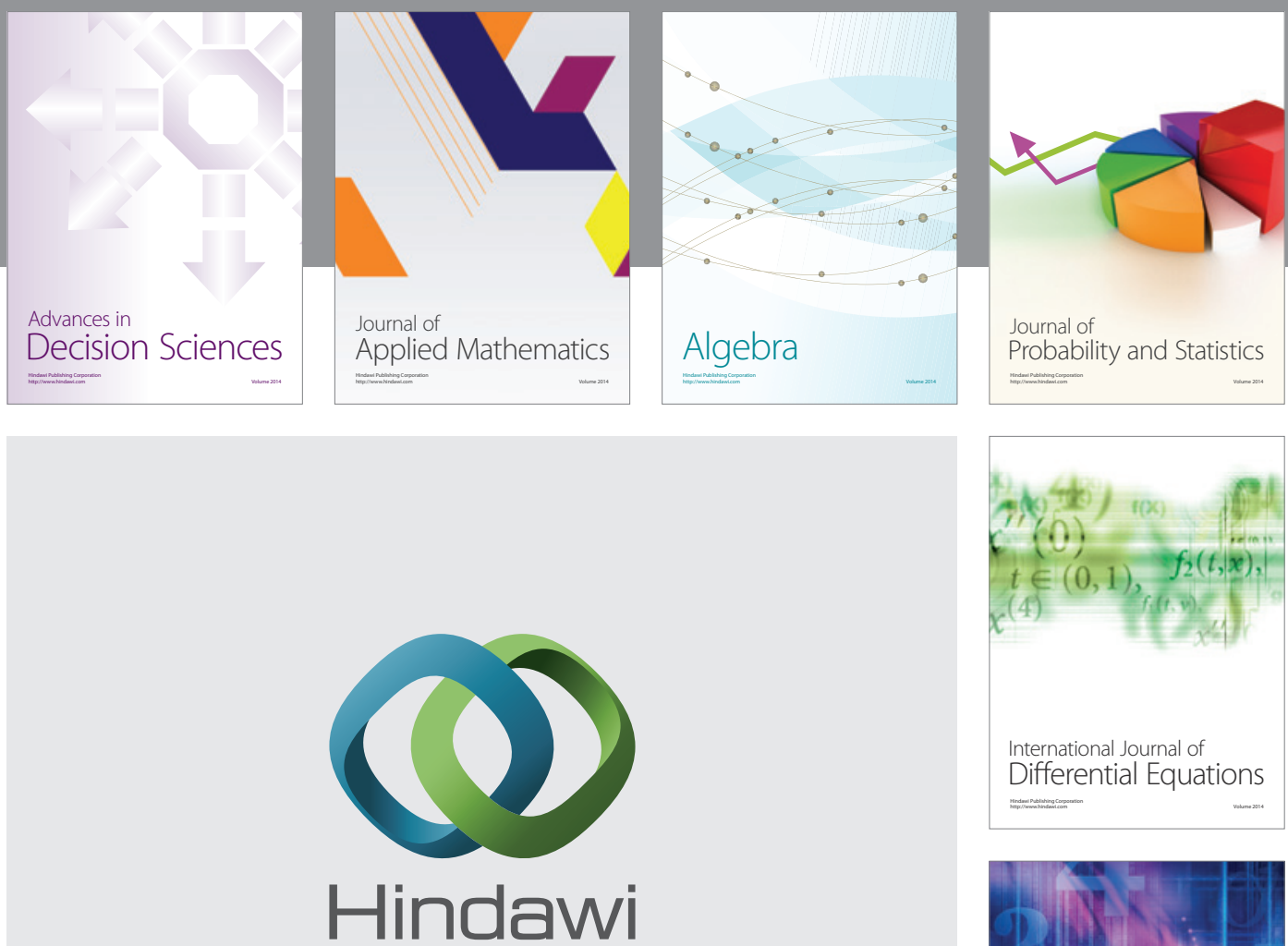

Submit your manuscripts at http://www.hindawi.com
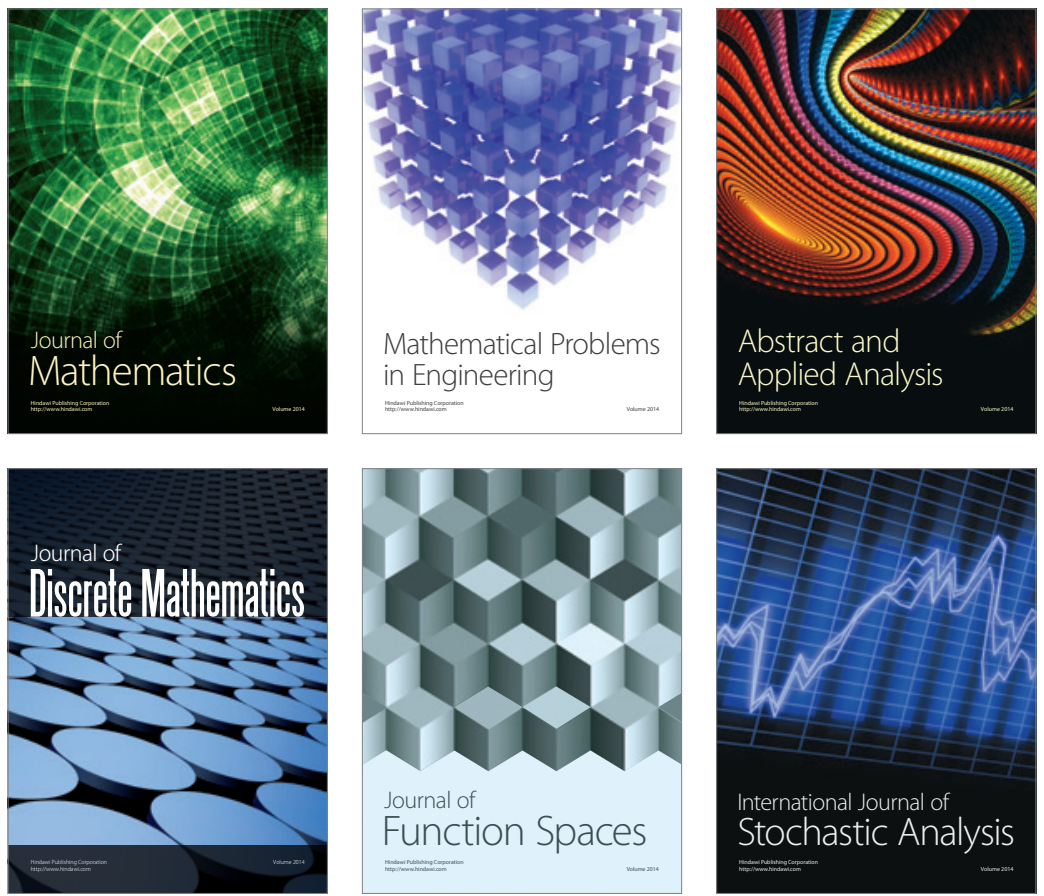

Journal of

Function Spaces

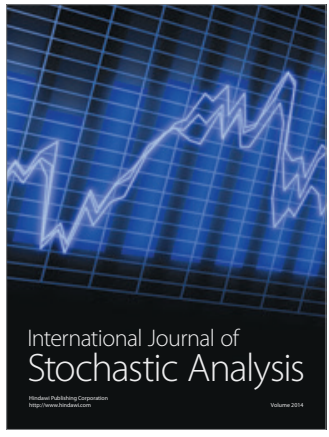

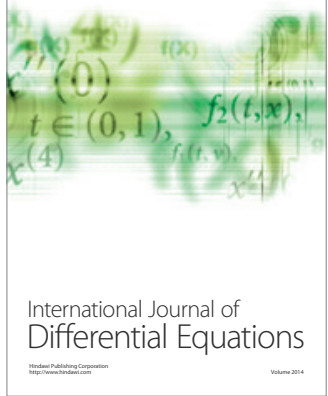
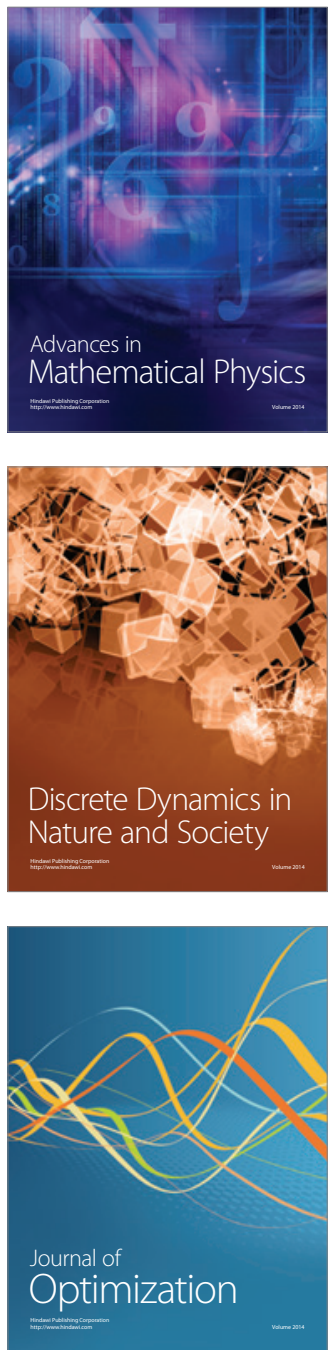